\title{
Multi-dimensional parametric assessment with IoT in acquaintance of digital pipeline
}

\author{
N. Suresh Kumar' ${ }^{1}$, Mallikharjuna Rao K ${ }^{2}$, Mahesh Kothuru ${ }^{3}$, Y.Narasimha Rao ${ }^{4}$ \\ ${ }^{1,3}$ GITAM Institute of Technology, GITAM (Deemed to be University), India \\ ${ }^{2}$ School of Computer Science and Engineering, VIT-AP University, India \\ ${ }^{4}$ Department of Computer Science and Engineering, QISCET, India
}

\begin{tabular}{l} 
Article Info \\
\hline Article history: \\
Received Apr 15, 2019 \\
Revised Jun 26, 2019 \\
Accepted Jul 6, 2019 \\
\hline Keywords: \\
Arduino \\
IoT \\
Parallel processing \\
Pipeline \\
Sensors \\
Virtual design
\end{tabular}

\begin{abstract}
In IoT researches are found millions of devices are connected in global digital infrastructure framed in network fashion. Most of the things on the network use cloud or central database to share their data and control signals to control and manage the devices on the IoT network. Devices on the network need fast traversing paths, to take correct decision in right time. The data synchronization between source and destination is very important in real time parameter maintenance. The multidimensional parametric evaluation of the environment or the surrounding area is facing problems with data synchronization and congestion in the sensor network. This will lead arrival of fault data at the destination. The fault data also leads a misconception at user end. In the present work pipeline integration is proposed to overcome the misconception and synchronization failures. The pipeline and Arduino UNO processor are integrated on Proteus simulation tool. Transducer nodes and the functionalities are analysed with pipeline.
\end{abstract}

Copyright $(2019$ Institute of Advanced Engineering and Science. All rights reserved.

\section{Corresponding Author:}

N. Suresh Kumar,

GITAM Institute of Technology,

GITAM (Deemed to be University),

Visakhapatnam, India.

Email: nskgitam2009@gmail.com

\section{INTRODUCTION}

The controlling of industrial appliances and home appliances are mainly depends on the current environment parameter measurements. The controlling of final measure-end either in an industry or at private building is mostly depends on multidimensional environmental parameters. For example, an IoT system is developed to control things of the manager's chamber located at work place. For instance, the manager would like to control the things present in the respective chamber before he/she arrives to the workplace from remote location. The remote location may be present when in busy traffic, or at home, or while travelling to the workplace. Managing the things before arriving the actual location will save the time and can get more accurate and desired results.

Similarly, in an industry the site engineer can steer the actuators located at hazardous unit from the personal chamber itself. The site engineer can watch the real time parameters from their chamber. In all these cases the sensors at hazardous places plays very important role and allow the field engineer to react before it became too late and in correct time. Hence, the transducers at the hazardous place must be quick enough to sense, convert and send the data to the engineer sitting at remote location. After receiving the data from hazardous unit the engineer sitting at control room can send commands/control signals to control the final measure ends at industrial appliances such as boiler, control valve, switching electric motors, relays, actuators, and traffic light signals, home appliances such as burglar alarm, air conditioner, gas valve, and 
triggering stove, and many more $[1,2]$. The definition of IoT elucidate, it is a global infrastructure for the information society enabling advanced services by interconnecting (physical and virtual) things based on, existing and evolving, interoperable information and communication technologies (adopted from ITU work on Internet of things, 2015).

In all the above applications the reaction of sensors and actuator at proper time is very important. In some scenarios a single sensor parameter can-not play vital role. The dependent sensors also plays important role along with the primary sensor. In the present paper the multi-dimensional environment is defined as at any point in the environment the parameters change due to few of the other physical parameters. For example the temperature in a room depends on several other parameters like previous room temperature, humidity, wind velocity, and number of persons present in that room. In smart intelligent system the controller can set the temperature based on other dependent parameters such as humidity, past temperature, strength of the air, and number of the persons. With all due respect the reading of multiple sensor measurement is one of the biggest challenges. Reading individual sensor reading and sending the readings for further processing to take decision for final control element is a tedious job. Manging final control element is dependent on multiple sensor outputs. Hence, the sensor outputs must reach the processor or final control element at target time. But, due to the limitation of number of ports at IoT device or due to synchronization problem there may be a chance of latency in data transmission or some data loss. Before arriving the next data at TxD pin of IoT the current data must pass through successfully although there is synchronization problem [3,4]. This situation arises with the problem that is jitter. The jitter due to synchronization problem creates severe problems in the data communication between IoT and destination such as client or cloud or final control element. This problem can be drastically decrease by introducing some intermediate buffers between IoT devices and destination.

Single Buffer scheme is simple to implement but it is mostly used for low data rates, and also increases the clock skew problem. In case of double buffered scheme wide range of data rates can be evaluated but there is a limitation to maintain lower signal strength [5]. Hence in the present work a pipeline technique is implemented to minimize data lag and misconception at the destination. All sensor parameters are fed to destination through pipeline inorder to minimize the data lag. So that, it further helps in reducing the misconception at the destination process. A simple linear pipeline with simple clock scheme is used to synchronize the data $[6,7]$.

The measure-end of the field where parameters need to be measured is generally sent to client for understand the situation and further controls the actuators at the field area. The data some-times directly send to the actuators to control the final control element for fast action. Sometimes an immediate action is needed to be implemented without waiting for any user command, in emergency situations such as when, gas sensor detects any leakage in closed chamber [8-10]. To understand the environmental situation and to control the things remotely from home or office, all sensors data must be synchronized with the client device. Before the data arrives to the final control element or the client device such as laptop or mobile phone, the data may be overwritten with the next data. This can be overcome with pipeline based IoT as shown in Figure 1.

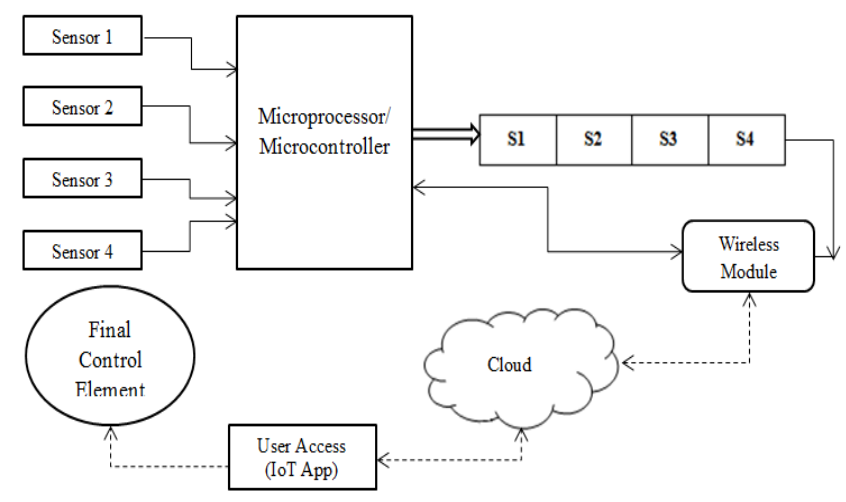

Figure 1. Framework of proposed architecture

The microprocessor or microcontroller system measures the parameters from several sensors and controls the final control element or actuator depending on processing of multiple parameters. In this case the sensory data can be arranged according to the programming sequence through pipeline. The pipeline also has an advantage of retaining the next parameter of the first sensor in the pipeline, while it is reading the first parameter from the next sensor. The framework of the proposed IoT system is shown as in Figure 1. 
The pipeline temporarily retains the previous data until it is fetched from the destination. The data pushed to client or actuator through pipeline. While the pipeline shifts the data to the destination, simultaneously it will fetch the next data. So there will be no time lag and optimizes the data fetching rate and processing rate [5].

\section{CIRCUIT DESCRIPTION}

In the present work temperature, humidity, rain drop sensor, and light detector are used to detect multiple parameters. The light detector is used to detect number of persons entering into the room; temperature sensor is used to provide current room temperature. Along with the current temperature value, and number of persons, the humidity also helps the user to govern the final control element. All the sensors are connected to the port pins of Arduino processor. The outputs are connected to Serial-In-Seral-Out (SISO) shift register. The SISO shift registers will pass the information to the destination. For, observation a LCD display is connected to the output of the shift registers. As the first register pushes the first data to the second register the first register will receive the next output data from the processor. Like that, it can hold 4 snippets at the same time. Hence memory dependency also reduced in this case. The data can also be saved into cloud or local-data-centre for retrieving the data in future. The pipeline final stage data can be sent to cloud with the help of ESP 8266. The basic components of IoT system shown in Figure 2 are explored in the following paragraphs [11-12].

- Humidity Sensor: The SHT10 is used for humidity measurement. This is belongs to SHT1X series, and it is used to measure both temperature and humidity. Both sensing and signal processing functional units are embedded onto a single unit chip. The important feature is that it has fast response time, good signal quality and insensitivity to external electromagnetic interferences. The package contains 4 use full pins and rest are not used and they left isolated. The ground pin and VDD pins are connected with 3-5V power supply. The data pin is bi-directional pin and SCK (serial clock) pin acting as input pin only. The SCK signal is used to establish handshaking signals between SHT10 and Arduino. The SCK signal will control the data operations at sensor interfacing. The DATA pin of SHT10 will act as transceiver. At the rising edge of the SCK, the command will be given to sensor. Command will enter into sensor only when DATA is valid. The DATA value will be changed after falling edge of SCK.

- Optical Sensor: The TSL251RD is used as optical sensor to convert light to voltage. In this work the light to voltage converter is used to detect number of persons entered into a closed room. It is a light detecting sensor has three active pins. One is GND pin and VDD pin is connected to power supply. The OUT pin produces output voltage when sensor detects light signal. This output voltage obtained at the OUT pin directly proportional to the intensity of the light radiated on the photodiode of the sensor.

- Temperature Sensor: The LM235 is used to sense the environmental temperature. It is a high precision temperature sensor. The output of the sensor is proportional to the absolute temperature. It has linear output and typical error is very small and almost less than to $1^{\circ} \mathrm{C}$ over $100^{\circ} \mathrm{C}$. The package contains three pins $\mathrm{V}+$, ADJ, and V-.

- Arduino Uno: In the present work Arduino Uno is opted to implement the current objective. The high performance RISC architecture of Arduino Uno board consists of several designs like microcontrollers, integrated memory, ADC, DAC, PWM channels, timers, serial ports, and input/output pins. In the current design 8-bit ATmega328 microcontroller based Arduino Uno device is selected to design the present system. The board can be functioned with external DC power supply. The 5V power supply may not produce constant $5 \mathrm{~V}$ and may have some fluctuations due to which the board may not support stable operations. The overload also causes some severe damage to the board. Hence, the recommended power supply is in between $6 \mathrm{~V}$ to $10 \mathrm{~V}$. It also supports 24 programmable $\mathrm{I} / \mathrm{O}$ multiplexed port pins. Here multiplexed pins indicate they carry both IO data and special control signals. It has $32 \mathrm{X} 8$ general purpose registers. It has $32 \mathrm{~KB}$ flash memory.

- LCD Display: In the present work TG13650 LCD display module is used to display the sensor readings. The LCD package contains 17 pins and can be operated in either 4-bit mode or 8-bit mode. It has one ground pin and one power supply pin operates at $+5 \mathrm{~V}$. The VR pin is power control register pin used to enable and disable internal voltage regulator circuit. $\overline{R D} \& \overline{W R}$ pins are used to read and write the data from command register and to write data into data register. The commands and data are sending to LCD module through D0 to D7 pins. In the present work only lower nibble is used to send the sensing data from pipeline. Connect $+5 \mathrm{~V}$ power supply between EL1 and EL2 pins to adjust the intensity.

- Pipeline: The digital pipeline plays very important and key role in synchronizing the data between microprocessor/microcontroller and data destination or final control element. In the process of data communication with the final control element or data storage device, it is a high responsibility of the IoT device to send the accurate data in requisite time period with high reliability. The measured data from

Multi-dimensional parametric assessment with IoT in acquaintance of digital pipeline (N. Suresh Kumar) 
the field are need to be carried to the destination in correct time before it become an out-time data. If the data reaches in correct time then only the system is able to take correct decision in right time. There are also some possibilities of some situations like the decision or prediction not only present on the present and previous immediacy data but also most likely depend on immediate next sensor measurement data. As the data fetching is a continuous process the data may not reach to the destination because of improper synchronization or mishandling of handshaking signals. There are also chances that the previous data is over written with the latest data before the previous data uploaded to the destination [13]. These problems can be overcome with pipeline technology. The pipeline is able to retain the data temporarily and allows the data to reach the destination through it [5, 7]. In the present circuit design as shown in the Figure 2 serial pipeline is connected at the Arduino output ports. Later parallel pipeline is interfaced at the individual output port pins (AD5-AD7) of Arduino processor. As the figure space does not allow to show here all the devices along with parallel pipeline in the system architecture, the parallel pipeline architecture is separately shown in Figure 3. An oscilloscope is connected across the pipeline to study the data rate and fetching style happening through the pipeline.

- Cloud: The necessity of cloud is high and became one of the key components in the present digitization world. In the present work a cloud is used to save sensors processed data. Thing Speak is one of the open source allows to store and retrieve the stored sensory data from various platforms. Thing Speak can be accessed using http protocols over the internet [14].

- Wi-Fi Model: Here ESP8266 is used to enable Wi-Fi model to communicate with the internet protocols. The ESP8266 is a Wi-Fi module contains TCP/IP protocol. The Wi-Fi module is sequentially configured with commands to enable network protocols.

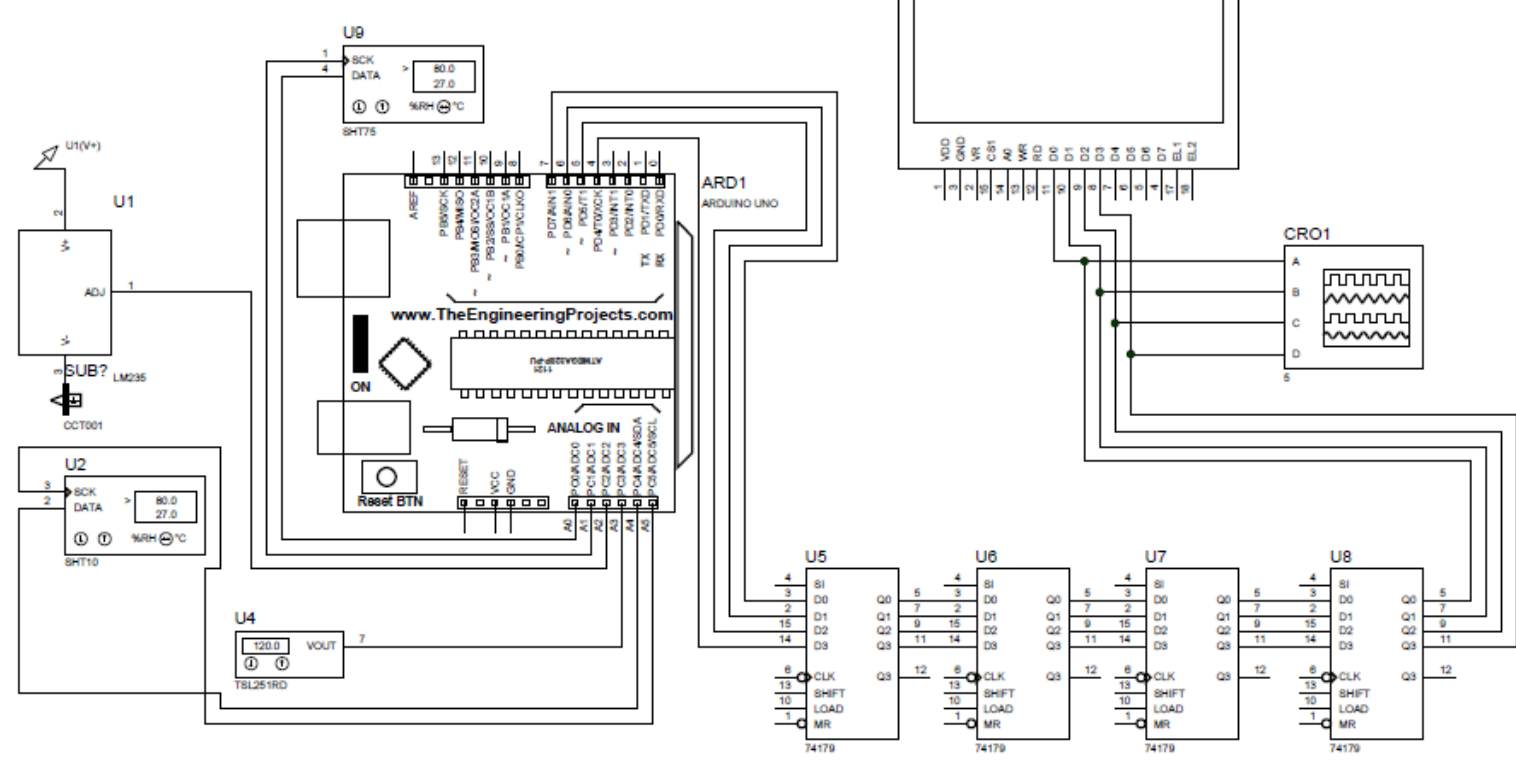

Figure 2. Pipeline based IoT system

\section{RELIABILITY OF THE DATA EMINENCE}

IoT become one of the most disruptive civilian technologies and develops a gigantic network in the universe by interconnecting of billions of peripherals by 2020 [9]. The IoT optimizes the automations in technological segments like Autonetics, Domotics, Robotization, Artificial Intelligence, Electronic Communication, Radio-Dynamics, Tele-mechanics [15, 16]. The IoT has already spread its wings and spreading drastically in many sectors such as, smart home, smart city, smart civil services, smart health, e-governance, logistics, smart merchandising, agriculture, food industry, automation, smart industrial applications, cyber security, and etc., [17, 18]. It is throwing many challenges and creating many opportunities for new generation in order to facilitate more flexible usage of IoT appliances. In all the sectors the main concern is with the scope of the data [19-21]. 
One of the prime challenge need to consider in IoT system is the scope of the data belong to the system [22-24]. The reliability of the data either in measurement or taking into consideration for controlling any actuator is mainly contingent on the life time of the data. Inorder to achieve a quality and consistent data it is very important to see the raw data is acquired in faster rate and processed in swift time. Proper analysis of massive raw data has to be through while multiple sensor readings play a key role in making a decision. While multiple sensor data are playing key role it is very important to retain the data before they complete the process. Hence an effective tool is required to retain the multiple sensory data. Rather than storing the data bytes at large storage disks like PROM and RAM, a simple temporary queue type device can satisfy the current problem. This queue can reduce the fetch time and enhances the decision speed.

In the current paper a data pipeline is used as queue to meet the purpose of retaining the data and fast fetching rate. The pipeline further improves the scope of the measuring parameters as their retaining time is improved for fraction of seconds [25]. For instance, on the public-road-traffic the sensors in the autonomous vehicles not only should observe the in-front vehicles movement but also the direction of movement on the road, i.e., whether it is moving from left to right or right left or moving in the same direction. Hence numerous sensors are required to interpret the direction of front object to the autonomous vehicle. Hence, from these data the autonomous vehicle can take appropriate decision in moving right direction. In this case the sensor data should arrive to the processing element with in fraction of time.

In another instance while a person travelling from office to his home and found that the journey is taking long time and hence, decides to operate home appliances before he arrives to the home. To switch on the geyser and set the time period, observe the water tank temperature, humidity in the environment at the home are some of the more prominent parameters need to be monitor and control. Similarly to open the windows at home the person should analyse the room temperature and percentage of odour occurrence before he controls the motors actuating the windows. In all the above cases the user decision depends on numerous physical parameters. Hence basically in situations like stochastic systems a new tool or technique is required in managing measurement of multiple sensor parameters. The pipeline technology is one of the best technique can be used to handle multiple parameters. In the proposed paper a four byte pipeline is interfaced with Arduino processor as shown in Figure 1. For serial pipeline four stages are used as the space complexity is high but, while coming to the parallel pipelining three stages are constructed. A single serial data pipeline may create bottleneck due to clock skew [4]. This can be overcome with parallel pipeline whose design is shown in Figure 3. The design contains three 3-stage pipelines all are acting simultaneously for realising enhanced fetching data rates.

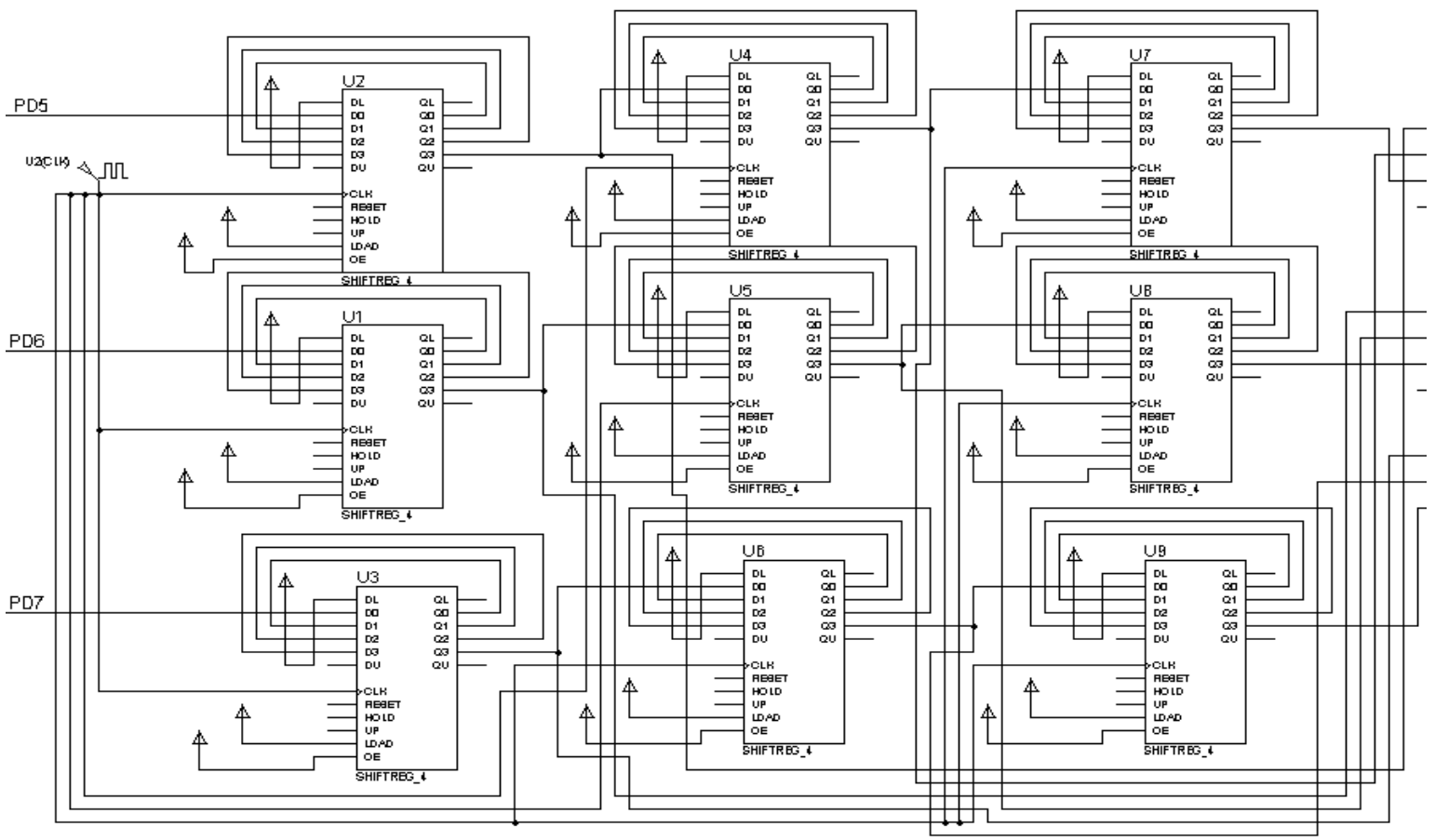

Figure 3. Three stage parallel pipeline used in fetching the arduino data 
In Figure 3 there are three stages of pipelines and are interfaced with port pins of Arduino processor. In the first row the first pipeline is connected with PD5 pin, second pipeline and third pipeline are interfaced with next port pins PD6 and PD7 respectively. All the clock pins of every stage of individual pipeline are synchronized with common clock pulse. A common clock pulse is connected so, that all the pipelines triggers simultaneously and receives the system outputs from the port pins. The output of each pipeline is out through Q3 output pin of last stage of pipeline. Each output pin (Q3) of last stage of each pipeline is connected with the individual virtual CROs (CRO1, CRO2, and CRO3). The Q3 of each pipeline is interfaced with virtual CROs for analysis of three stage pipeline. These simulation results are shown in Figure 5.

\section{RESULT}

In this section the functional description and corresponding simulation results obtained in the Proteus VSM tool are presented. The simulation results of parallel pipeline obtained in Proteus tool are shown in Figure 4. The three Oscilloscopes CRO1, CRO2, and CRO3 are connected to the last stage of each pipeline. The first pipeline, second pipeline, and third pipeline outputs are individually connected with CRO1, CRO2, and CRO3 respectively. The Figure 4 contains three tabs showing that the results obtained in three CROs. The tab name with "Digital Oscilloscope - CRO1" represents the output obtained at first pipeline connected with PD5 pin. The same is produced for other two pipelines connected at PD6 and PD7 pins. The pulses shown in Figure 5 with yellow colour pulse depicts the output of pipeline first stage, the blue colour pulse is reflecting to the output of pipeline second stage, and the pink colour pulse represents the output of third stage pipeline.

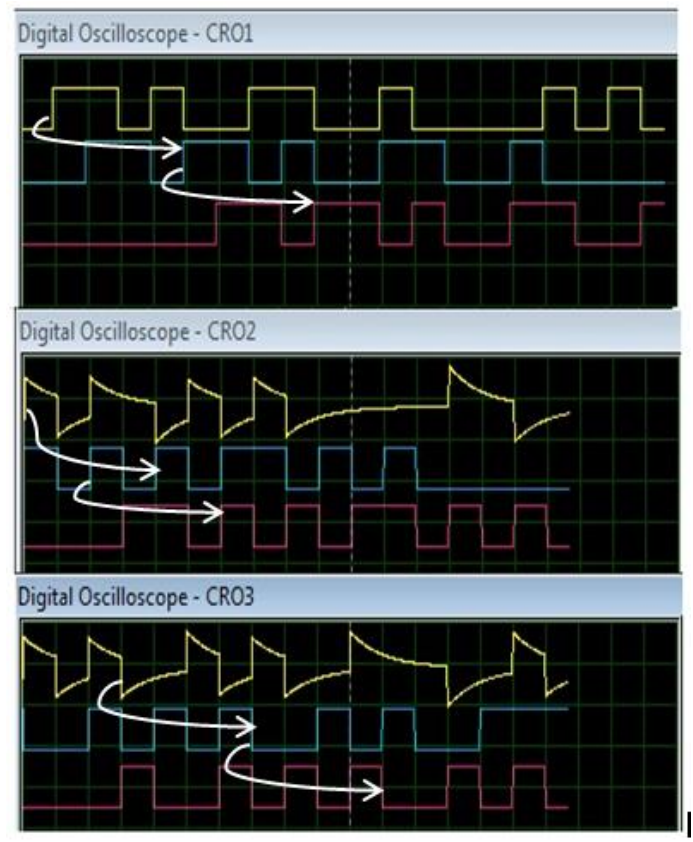

Figure 4. Simulation results

The figures are clearly stating that while third stage is producing an output pulse at the same time when the second stage and first stage of the pipeline are reading next consecutive pulses. Figure 5 is showing that in the first clock pule only F1 entered into the pipeline. The stage produces output after third clock pulse. At third clock pulse the three fetched codes are entered into the pipeline simultaneously. The F1, F2, and F3 will retain in the same stage until next clock pulse triggers. After the next clock pulse triggering the F1 comes out and goes to the next module in the system. At the same time F1 pushes F2. F2 pushed into the third stage and F1 enters into the second stage. Now F4 is fetched and enters into the first stage. The detailed operation is presented in Figure 5. In Figure 4 the white arrow marks drawn in the output of CRO1 stating the displacement of the pulses from one stage to next stage in the pipeline. That is the propagation of data waves in different stages of pipeline are representing with white arrow. The same is embodied in all other pipelines and the respective outputs are shown in CRO2 and CRO3 of Figure 4. 


\begin{tabular}{ll|l|lll} 
F1 & F2 & F3 & F4 & F5 & F6 \\
\hline & F1 & F2 & F3 & F4 & F5 \\
& & F1 & F2 & F3 & F4 \\
\hline
\end{tabular}

Figure 5. Fetching pulses through three stage pipeline

\section{CONCLUSION}

In the present work different sensor measurements are evaluated and configured with IoT modules. The parameters are stored in the cloud through pipeline. The multiple measure-end values which are playing an important role in deciding required field value, is obtained with the help of parallel processing element pipeline. Three stages of Pipeline are designed, implemented and their behaviour is analysed with virtual simulator Proteus. Three stage pipelines are interfaced with IoT module Arduino Uno processor. The pipeline clock design can be further optimized by implementing wave, mesochronous and hybrid clock schemes to minimize clock skew in the pipeline stages.

\section{REFERENCES}

[1] L. Atzori et al., "The internet of things: A survey," Comput. Netw., vol. 54, no. 15. pp. 2787-2805. 2010.

[2] Andrea Zanella et al., "Internet of Things for Smart Cities," IEEE Internet of things journal, vol. 1, no. 1, pp. 22-32, Feb 2014.

[3] N. Suresh Kumar et al., "A New Method to Enhance Performance of Digital Frequency Measurement and Minimize the Clock Skew," IEEE Sensor Journal, vol. 11, no. 6, 2011.

[4] N. Suresh Kumar N et al., "Digital frequency meter using DMA Terminal Count Stop method," IJET, vol. 2, no. 1, pp. 34-37, 2010.

[5] Milan Prokin, "Double Buffered Wide-Range Frequency Measurement Method for Digital Tachometers," IEEE Transactions on instrumentation and measurement, vol. 40, no. 3, Jun 1991.

[6] N. Suresh Kumar et al., "Effect of performance of pipeline with Interrupt Logic," Iject, vol. 2, no. 3, Sep 2011.

[7] L. Cotten, "Maximum rate pipelined systems," in Proc. AFIPS Spring Joint Comput. Conf., 1969.

[8] Hongbo jiang et al., "Smart Home Based on WiFi Sensing: A Survey," IEEE Access, Digital Object Identifier 10.1109/ACCESS.2018.2812887, vol. 6, 2018.

[9] P. Jagannadha Rao et al., "Detection of Rain Fall and Wind Direction using Wireless Mobile Multi Node Energy Efficient Sensor Network," International Journal of Applied Information Systems (IJAIS), vol. 3, no. 9, Feb 2012.

[10] Chi-Sheng Shih et al., "Designing CPS/IoT applications for smart buildings and cities," IET Cyber-Physical Systems: Theory \& Applications, vol. 1, no. 1.pp. 3-12, 2016.

[11] Grzegorz Lehmann et al., "A 3-Layer Architecture for Smart Environment Models," 8th IEEE International Conference on Pervasive Computing and Communications Workshops. Apr 2010.

[12] Ganche, I., Ji, Z., O’Droma, M., “A generic IoT architecture for smart cities,” In: Proceedings of 25th IET Irish Signals \& Systems Conference, 2013, pp. 196-199.

[13] N. Suresh Kumar et al. "Two Way Clock Scheme in Pipeline to Minimize the Clock Skew," GJCST, vol. 10, no. 9, Sep 2010.

[14] Kuchi N S S S S Utpala et al., "Authenticated IoT Based Online Smart Parking System with Cloud,” Pramana Research Journal, vol. 9, no. 4, 2019.

[15] Chi-Sheng Shih et al., "Designing CPS/IoT applications for smart buildings and cities," IET Cyber-Physical Systems: Theory \& Applications, vol. 1, no. 1, pp. 3-12, 2016.

[16] Stankovic, J.A., "Research directions for the internet of things," IEEE Internet of Things Journal, vol 1, no. 1, pp. 3-9, 2014.

[17] Joshua E. Siegel et al., "The Future Internet of Things: Secure, Efficient, and Model-Based," IEEE Internet of things journal, vol. 5, no. 4, pp. 2386-2398, Aug 2018.

[18] L. Atzori, et al., "The Internet of Things: A survey," Comput. Netw., vol. 54, no. 15, pp. 2787-2805, Oct 2010.

[19] C. C. Aggarwal et al., "The Internet of Things: A Survey From the Data-Centric Perspective," Boston, MA, USA: Springer, pp. 383-428, 2013.

[20] Q. Wu et al., "Cognitive Internet of Things: A new paradigm beyond connection," IEEE Internet of Things Journal, vol. 1, no. 2, pp. 129-143, Apr 2014.

[21] Claude Castelluccia, "Efficient and Provably Secure Aggregation of Encrypted Data in Wireless Sensor Networks," ACM Transactions on Sensor Networks, vol. 5, no. 3, May 2009.

[22] Chang-Su Ryu, et al., "Monitoring System for Integrated Management of IoT-based Home Network," International Journal of Electrical and Computer Engineering (IJECE), vol. 6, no. 1, pp. 375-380, Feb 2016.

[23] Zulnazim Dzulkurnain et al., "Internet of things (IoT) based traffic management \& routing solution for parking space," Indonesian Journal of Electrical Engineering And Computer Science (IJEECS), vol. 15, no. 1, pp. 336-345, 2019.

[24] C.S. Ryu and H.W. Hur, "Home Network Monito ring System Based on Internet of Things," 2015 International Conference on Future Informa tion \& Communication Engineering. ICFICE2015, 2015, pp. 79-82.

Multi-dimensional parametric assessment with IoT in acquaintance of digital pipeline (N. Suresh Kumar) 
[25] Suresh Kumar N., Rama Koti Reddy D.V., "Optimizing Technique to Improve the Speed of Data Throughput Through Pipeline," In: Satapathy S., Mandal J., Udgata S., Bhateja V. (eds) Information Systems Design and Intelligent Applications. Advances in Intelligent Systems and Computing, Springer, New Delhi, vol. 435, 2016.

\section{BIOGRAPHIES OF AUTHORS}

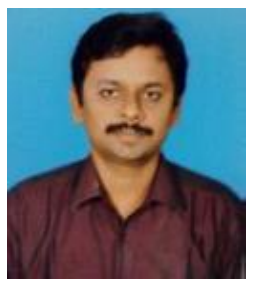

Dr. N. Suresh Kumar has received bachelor of engineering in 2001 from Berhampur University, and Master of Technology in 2005 from Allahabad Institute of Deemed University. He has obtained Ph.D. on Reconfigurable Computing Systems from Andhra University. His research of interest is parallel processing, Natural Language Processing, Reconfigurable Computing Systems, and Computerised Process Control Measurement. He is currently working as Assistant professor in GITAM University, Visakhapatnam, India. During his 16 years of experience he occupied different positions in academic and administration. He also interested in developing and adopting new teaching methodologies in class room. He has published few international papers on Information Communication Technology. He has published research papers in various refereed National and International Conferences and journals. The author has reviewed few of the conference papers and also acting as editorial board member in reputed journals.

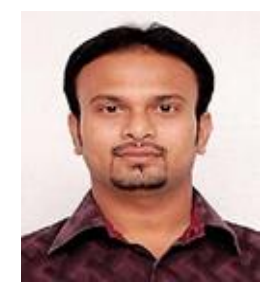

Dr. Mallikharjuna Rao K., currently, working as Assistant Professor Sr. Grade1 in School of Computer Science and Engineering, VIT-AP University, Amaravati, Andhra Pradesh, India. He obtained Bachelor's degree B.Tech in Information Science and Technology Engineering from Acharya Nagarjuna University, Guntur, Andhra Pradesh, India. Then he obtained Master's degree M.Tech in Computer Science from Jawaharlal Nehru Technological

University Hyderabad, India and obtaind doctoral degree Ph.D. in Computer Science and Engineering from Jawaharlal Nehru Technological University Hyderabad, India. He has more than $14+$ years of teaching experience in various engineering Institutions in A.P. and Telangana. His specializations include Software Reliability, Software Testing, Artificial Intelligence, Parallel processors, and Evolutionary Computing. Email id: rao.mkrao@ gmail.com.

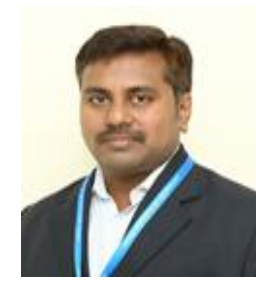

Mahesh Kothuru, He received his Engineering from MVGR College of Engg., Department of Computer Science and Engineering in 2009, M.Tech. from the Andhra University, Department of Computer Science and Systems Engineering in 2014, Started his research work in GITAM since 2018, where he now works as Assistant Professor. His research area is on Computer Networks, Cloud Computing and Internet of Things.

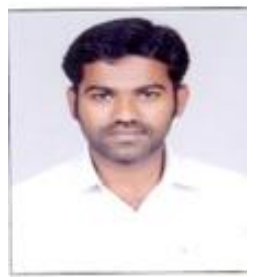

Dr. Y. Narasimha Rao has received B.E from Jawaharlal Nehru Technological University (Hyderabad,India) and M.Tech from Acharya Nagarjuna University (Guntur,India) and has completed his Ph.D from Andhra University(Visakhapatnam,India).He is dedicated to teaching field from the last 15 years and currently working as Head of CSE\&IT in QIS College of Engineering and Technology(Autonomous), Ongole, Andhra Pradesh, India 